\title{
Molecular Evidence of Spirometra erinaceieuropaei in Asian Wild Frogs (Rana rugulosa) from Banyuwangi City, Indonesia
}

\author{
Aditya Yudhana ${ }^{1 *}$, Ratih Novita Praja ${ }^{2}$, Maya Nurwartanti Yunita ${ }^{3}$ and Dhandy Koesoemo Wardhana ${ }^{4}$ \\ ${ }^{I}$ Department of Parasitology, Faculty of Veterinary Medicine, Universitas Airlangga, Indonesia \\ ${ }^{2}$ Department of Microbiology, Faculty of Veterinary Medicine, Universitas Airlangga, Indonesia \\ ${ }^{3}$ Department of Pathology, Faculty of Veterinary Medicine, Universitas Airlangga, Indonesia \\ ${ }^{4}$ Department of Veterinary Public Health, Faculty of Veterinary Medicine, Universitas Airlangga, Indonesia \\ *Corresponding author's Email: adityayudhana@fkh.unair.ac.id; (DORCiD: 0000-0001-9664-6380
}

\begin{abstract}
The tapeworm Spirometra erinaceieuropaei is the most frequent species found in wild frogs and causing a serious parasitic zoonosis known as sparganosis. This study aimed to provide molecular evidence of spargana collected from wild frogs which are used as food and contribute to providing important implications for the prevention and control of sparganosis. A total of 185 Asian wild frog (Rana rugulosa) samples were selected from food markets in Banyuwangi City, Indonesia. Molecular identification based on spargana that were collected and coding gene of mitochondrial cytochrome c oxidase 1 ( coxl) using Polymerase Chain Reaction (PCR) method. Spargana were found in $9.1 \%(17 / 185)$ of the frogs and PCR analysis results identified all specimens belonging to the species $S$. erinaceieuropaei, therefore indicated that $S$. erinaceieuropaei is the major causative agent of sparganosis from frogs which sold as food in markets. These findings can be useful to the molecular diagnosis and control of Spirometra infections in humans and animals.
\end{abstract}

Keywords: Asian wild frog, Rana rugulosa, Sparganosis, Spirometra erinaceieuropaei.

\section{INTRODUCTION}

The species of the genus Spirometra, including $S$. erinaceieuropaei (S. erinacei), S. decipiens, S. mansoni, S. ranarum, and $S$. mansonoides are all intestinal parasites of canine and feline hosts (Kavana et al., 2015). The main characters in differentiation of species are the spirally coiled uterus of S. erinaceieuropaei and S. decipiens (Jeon et al., 2015). The life cycle of these parasites require 2 different intermediate hosts; the fresh water cyclops as the first intermediate host, and vertebrates such as amphibians and reptiles as the second intermediate hosts. The procercoids develop in cyclops, and the plerocercoids (spargana) develop in frogs or snakes and cause sparganosis in humans (Wiwanitkit, 2005). The tapeworm Spirometra erinaceieuropae is the most important species of the genus Spirometra tapeworms (Nakao et al., 2000). Its plerocercoid larvae (spargana) can lodge in the subcutaneous tissues and sometimes invade the abdominal cavity, eye, and central nervous system of humans causing a serious parasitic zoonosis known as sparganosis (Nithiuthai et al., 2004; Cui et al., 2011).

Sparganosis is a parasitic zoonosis endemic in Asia, Europe, and North America (Kondzior et al., 2018; Sahoo et al., 2018; Scholz et al., 2019). Humans can be infected through the consumption of contaminated water or meat from intermediate hosts or through topical application of raw, contaminated poultices to eyes and open wounds (Jeon et al., 2015; Hong et al., 2016). After entry into humans, the plerocercoid larvae (spargana) migrate to different anatomic locations, where they cause space-occupying lesions as they develop into adults. The sites spargana migrate to include skin and soft tissues, muscles, visceral organs, and the central nervous system. Clinical symptoms range from asymptomatic/mild (e.g., subcutaneous swelling) to severe (e.g., seizure and hemiparesis) depending on the site and size of lesions (Liu et al., 2015).

Sparganosis is an emerging zoonotic disease and public health challenge in Asia, potentially because of the practice of consuming wild frog meat, which is a delicacy in Indonesia (Prasetyo and Safitri, 2019). Although human sparganosis is sporadically distributed around the world, it is most frequently reported in East and Southeast Asia (Qiu and Qiu, 2009). According to a 2009 survey, more than $25 \%$ of the local wild frogs in China were infected with spargana (Li et al., 2009). Another recent report showed 104 cases of human sparganosis in China from 2000 to 2006, and more than half, or $53.9 \%$, of cases were caused by eating snakes or frogs (Wu et al., 2007). In Thailand, over 60 sparganosis cases 
have been reported since 1943, and the patients were almost exclusively infected with $S$. erinaceieuropaei, with only few cases of S. proliferum infections (Anantaphruti et al., 2011; Boonyasiri et al, 2014).

In Indonesia, few cases of Spirometra infection have been reported from snakes in Sidoarjo and Mojokerto with total prevalence 68\% and 50.85\% respectively (Pranashinta et al., 2017; Yudhana et al., 2019). There have been several reports of molecular evidences for the detection of spargana infection in frogs (Jongthawin et al., 2014; Zhang et al., 2015). However, there is no molecular evidence of the parasite species in frogs in Indonesia. The aim of the present study is to provide molecular evidences of spargana collected from wild animals which used as food and consumption in Banyuwangi, East Java Province, Indonesia. These results may contribute to identify the sources of infection, which provide important implication for prevention and control of sparganosis in these areas. Moreover, we designed our analysis based on coding gene of mitochondrial cytochrome c oxidase 1 gene (coxl) of spagarna isolates from Asian wild frogs, Rana rugulosa from Banyuwangi, East Java Province, Indonesia.

\section{MATERIALS AND METHODS}

\section{Ethical approval}

This study was conducted with permission from the local agriculture department in East Java Province, Indonesia. This study was reviewed and approved by the Animal Care and Use Committee of Faculty of Veterinary Medicine, Universitas Airlangga, Indonesia No.1.KE.190.11.2019.

\section{Parasite samples}

A total of 185 living frog specimens (Rana rugulosa), commonly known as Chinese edible frog or East Asian wild frog were obtained from five food markets located around the Banyuwangi City (Central, West, East, North, and South parts) East Java Province, Indonesia (114.369227 Longitude and -8.219233 Latitude).

\section{Sparganum collection}

The presence of spargana in frogs was examined according to the methods of Ooi et al. (2000). The frogs were euthanized using ethyl-ether anesthesia, weighed, and skinned. The muscles and subcutaneous tissues were carefully observed for the presence of spargana by eyes. Then, the spargana were removed from the muscles or subcutaneous tissues and put in a Petri dish containing physiological saline to observe their movement. The number of spargana collected from each infected frog were counted to estimate the intensity of sparganum infection.

\section{DNA extraction and amplification}

Total genomic DNA was extracted from individual plerocercoid sample using the extraction kit (NucleoSpin ${ }^{\circledR}$ Tissue, Macherey-Nagel, Germany) following the manufacturer's protocol. A partial sequence of coxl was amplified using the primers Se658-F (5'-TTT GAT CCT TTG GGT GGT GG-3') and Se1124-R (5'-ACC ACA AAC CAC GTG TCA TG-3'), which were designed from the coxl gene of S. erinaceieuropaei (GenBank accession no. AB369250) (Boonyasiri et al., 2013). PCR was performed in a $25 \mu \mathrm{l}$ of reaction volume containing $10 \mathrm{ng}$ of DNA, $2.5 \mu \mathrm{l}$ of 10X FastStart High Fidelity Reaction buffer (Roche, Mannheim, Germany), $18 \mathrm{mM} \mathrm{MgCl}, 200 \mu \mathrm{M}$ dNTPs, $0.2 \mu \mathrm{M}$ each primer (Invitrogen, Carlsbad, CA), and 0.625 U FastStart High Fidelity Enzyme Blend (Roche). Thermocybcling conditions (conducted in GeneAmp PCR System 9700, Applied Biosystems, Singapore) were as follows: $94^{\circ} \mathrm{C}$ for 5 minutes; 35 cycles of $95^{\circ} \mathrm{C}$ for 30 seconds, $59^{\circ} \mathrm{C}$ for 30 seconds, and $72^{\circ} \mathrm{C}$ for 45 seconds; with a final step at $72^{\circ} \mathrm{C}$ for 10 minutes. For each PCR experiment, a negative (no DNA) and an amplicons were separated by $1 \%$ agarose gel-electrophoresis.

\section{RESULTS}

Spirometra spargana were identified in 9.1\% (17/185) of the frogs (Table 1). These spargana were 1-20 cm in long and $1-1.5 \mathrm{~mm}$ in wide. The prevalence of sparganum infection ranged from $3.0 \%$ to $13.6 \%$. Most host samples were infected by several spargana, and 1-3 sparganum was selected from each host sample (Table 1). Moreover, a total of 12 parasite samples were used for molecular identification. Partial sequences of the coxl gene were successfully amplified for each sample which shows positive bands at 467 bp (Figure 1).

PCR analysis results identified all 12 individual spargana specimens belonging to the species $S$. erinaceieuropaei. This study therefore indicated that $S$. erinaceieuropaei is the major causative agent of sparganosis from frogs which sold as street food, particularly in Banyuwangi City, Indonesia. 
Table 1. Spirometra and host samples from food markets in Banyuwangi City, Indonesia in 2019.

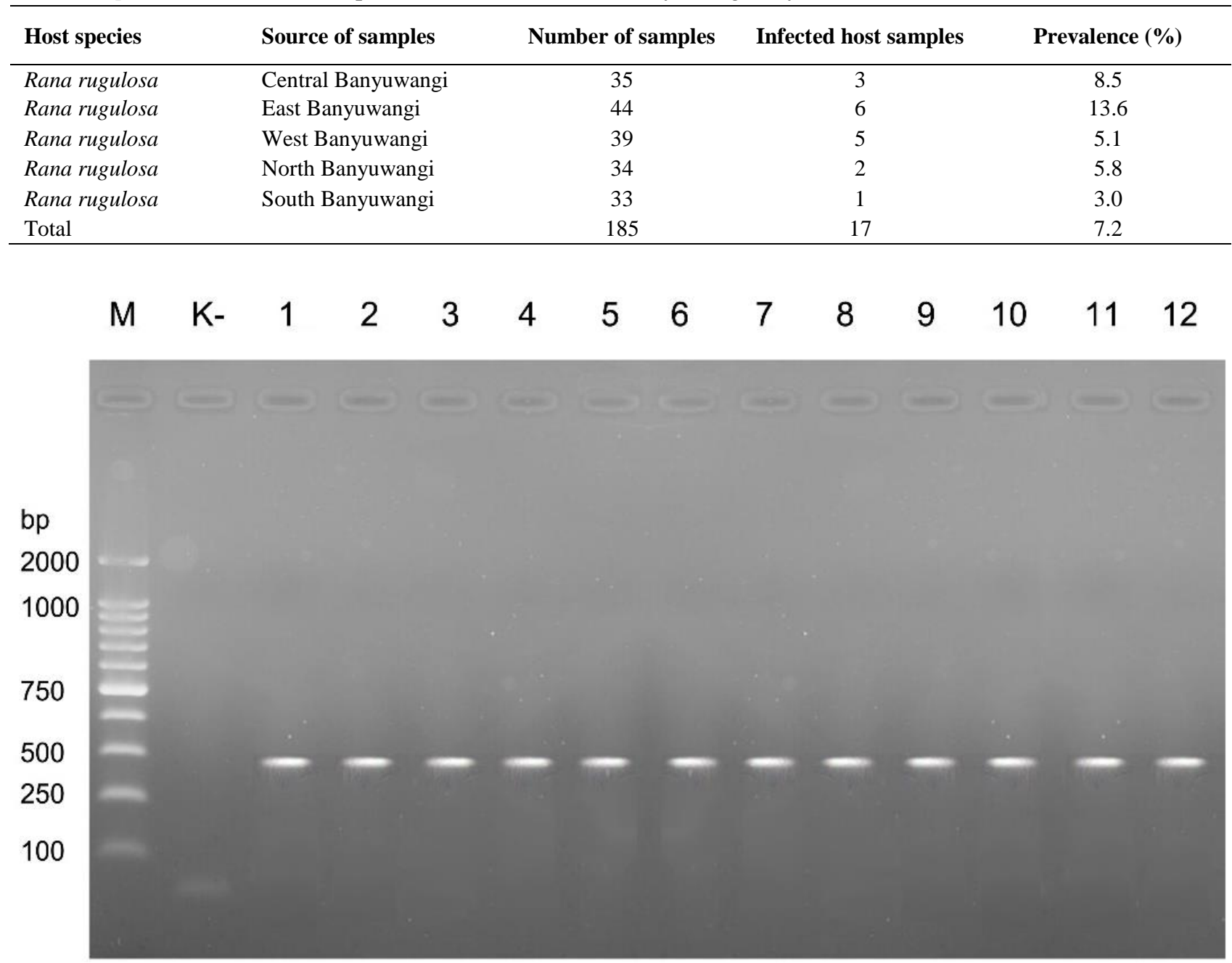

Figure 1. DNA visualization of coxl gene of Spirometra erinaceieuropaei in polymerase chain reaction products. Lane $\mathrm{M}=1: 100 \mathrm{bp}$ molecular weight standard, $\mathrm{K}-=$ Control negative.

\section{DISCUSSION}

Sparganosis is a food-borne infection caused by the migrating plerocercoid larvae of the tapeworm Spirometra spp. Frogs and snakes are the natural intermediate hosts for these parasites (Cui et al., 2011; Wang et al., 2011). In Chinese cuisine, which also sold in Indonesia, the bushmeat (meat of wild animals used as food) of frog and snake have an important place. The general public like this delicious bushmeat, but it poses as the potential risk of acquiring sparganosis. Spargana of S. erinaceieuropaei have also been found in many species of wild frogs, including Rana nigromaculata, R. limnocharis, R. temporaria and Bufo gargarizans (Liu et al, 2010; Wei et al., 2014). In a survey of Australian amphibians, S. erinaceieuropaei also was found in a variety of tree frogs, including Litoria caerulea, L. aurea, L. gracilenta, and L. peronii (Berger et al., 2009). There are several species in the genus Spirometra that can cause the disease, and the spargana of these species are often found in frogs, also there have been more than 1,700 global reports of sparganosis published (Liu et al., 2015).

Previous studies in East Java Province, Indonesia the prevalence of spargana was surveyed, but accurate species identification work was not done (Pranashinta et al., 2017; Yudhana et al., 2019).

It seems to the present study is the first report of $S$. erinaceieuropaei infection in frogs ( $R$. rugulosa) from Banyuwangi City, Indonesia. Genetic diversity among S. erinaceieuropaei specimens from neighboring countries was discovered, and such studies should be extended in order to obtain a more complete understanding of the molecular identification of this parasite in the Asian regions. Molecular taxonomy methods based on suitable markers are well documented for identification of a group of morphologically similar parasites. These markers, such as coxl have been used previously for the identification of S. erinaceieuropaei (Zhu et al., 2002; Okamoto et al., 2007; Liu et al., 2010; Wang et al., 2011; Liu et al., 2012; Wei et al., 2014).

In the present study, phylogenetic relationship of tapeworms within the Diphyllobothriidae family, based on partial coxl sequences clearly distinguished the genus Spirometra from Diphyllobothrium because of identified the spargana samples as S. erinaceieuropaei. Additionally, molecular identification studies from Japan (Okamoto et al., 2007) and 
India (Sahoo et al., 2018) of $S$. erinaceieuropaei obtained in dogs found similar variations in coxl sequences. Future study with a larger number of samples from various localities is needed in order to clarify the genetic diversity of $S$. erinaceieuropaei in Indonesia.

\section{CONCLUSION}

In present study indicated all spargana species of frogs (Rana rugulosa) which sold in food markets around Banyuwangi City, East Java Province, Indonesia were confirmed as S. erinaceieuropaei with $9.1 \%(17 / 185)$ prevalence rate. The results of this study can help to control of Spirometra infections in humans and animals. Additionally, we also propose the control measures for Sparganosis such as periodic inspection of S. erinaceieuropaei infection in frogs in markets and farms are necessary and scientific propaganda should be carried out by the local governments to inform the food restriction of wild caught frogs generally.

\section{Acknowledgments}

The authors would like to thank all members of Reptile Rescue Community and Laboratory assistant in the Department of Veterinary Parasitology for their help during the investigation and paper preparation. This work was supported financially by the Penelitian Internal Universitas Airlangga, Indonesia (no.1408/UN3/2019).

\section{DECLARATIONS}

\section{Authors' contributions}

AY is a supervised and project leader. RNP carried out the collection of frog samples, and MNY carried out dissection of frog samples. DKW is a data analysis and help with collected samples. All authors contributed to the drafting and revision of the manuscript. All authors read and approved the final manuscript.

\section{Competing interests}

The authors declare that they have no competing interests.

\section{REFERENCES}

Anantaphruti MT, Nawa Y and Vanvanitchai Y (2011). Human sparganosis in Thailand: an overview. Acta Tropica, 118: 171-176. DOI: https://doi.org/10.1016/j.actatropica.2011.03.011

Berger L, Skerratt LF, Zhu XQ, Young S and Speare R (2009). Severe sparganosis in Australian tree frogs. Journal of Wildlife Disease, 45: 921-929. DOI: https://doi.org/10.7589/0090-3558-45.4.921

Boonyasiri A, Cheunsuchon P, Srirabheebhat P, Yamasaki H, Maleewong W and Intapan PM (2013). Sparganosis presenting as cauda equina syndrome with molecular identification of the parasite in tissue sections. Korean Journal of Parasitology, 51: 739-42. DOI: https://doi.org/10.3347/kjp.2013.51.6.739

Boonyasiri A, Cheunsuchon P and Suputtamongkol Y (2014). Nine human sparganosis cases in Thailand with molecular identification of causative parasite species. The American Journal of Tropical Medicine and Hygiene, 91: 389-393. DOI: https://doi.org/10.4269/ajtmh.14-0178

Cui J, Li N, Wang ZQ, Jiang P and Lin XM (2011). Serodiagnosis of experimental sparganum infections of mice and human spargan osis by ELISA using ES antigens of Spirometra mansoni spargana. Parasitology Research, 108: 1551-1556. DOI: https://doi.org/10.1007/s00436-010-2206-2

Hong Q, Feng JP, Liu HJ, Li XM, Gong LR, Yang Z, Yang WM, Liang XF, Zheng RJ, Cui ZC, Wang WL and Chen DX (2016). Prevalence of Spirometra mansoni in dogs, cats, and frogs and its medical relevance in Guangzhou, China. International Journal of Infectious Disease, 53:4145. DOI: https://doi.org/10.1016/j.ijid.2016.10.013

Jeon HK, Park H, Lee D, Choe S, Kim KH, Huh S, Sohn WM, Chai JY and Eom KS (2015). Human Infections with Spirometra decipiens Plerocercoids Identified by Morphologic and Genetic Analyses in Korea. Korean Journal of Parasitology, 53 (3): 299-305. DOI: https://doi.org/10.3347/kjp.2015.53.3.299

Jongthawin J, Intapan PM, Sanpool O, Sadaow L, Laymanivong S, Thanchomnang T and Maleewong W (2014). Molecular evidence of Spirometra erinaceieuropaei infection in snakes Ptyas korros from Lao PDR and Thailand and Frogs Hoplobatrachus rugulosus from Myanmar. Southeast Asian Journal of Tropical Medicine and Public Health, 45(6): 1271-1278. Available at: https://www.seameo.org

Kavana NJ, Kassuku AA and Kasanga CJ (2015). Incubation of Spirometra eggs at laboratory conditions by Modified Harada-Mori method. Huria Journal, 19: 29-36. Available at: https://www.ajol.info

Kondzior E, Tokarska M, Kowalczyk R, Ruczynska I, Sobocinski W and Kolodziej-Sobocinska M (2018). The first case of genetically confirmed sparganosis (Spirometra erinaceieuropaei) in European reptiles. Parasitology Research, 117: 3659-3662. DOI: https://doi.org/10.1007/s00436018-6079-0

Liu W, Zhao GH and Tan MY (2010). Survey of Spirometra erinaceieuropaei spargana infection in the frog Rana nigromaculata of the Hunan Province of China. Veterinary Parasitology, 173: 152-156. DOI: https://doi.org/10.1016/j.vetpar.2010.06.005

Liu W, Liu GH and Li F (2012). Sequence variability in three mitochondrial DNA regions of Spirometra erinaceieuropaei spargana of human and animal health significance. Journal of Helminthology, 86: 271-275. DOI: https://doi.org/10.7150/ijbs.4096

Liu Q, Li MW, Wang ZD, Zhao GH and Zhu XQ (2015). Human sparganosis, a neglected food borne zoonosis. The Lancet Infectious Disease, 15:1226-1235. DOI: https://doi.org/10.1016/S1473-3099(15)00133-4

Li MW, Lin HY, Xie WT, Gao MJ, Huang ZW and Wu JP (2009). Enzootic sparganosis in Guangdong, People's Republic of China. Emerging Infectious Disease, 15:1317-1318. DOI: https://doi.org/10.3201/eid1508.090099

Nakao M, Sako Y, Yokoyama N, Fukunaga M and Ito A (2000). Mitochondrial genetic code in cestodes. Molecular and Biochemical Parasitology, 111: 415-424. DOI: https://doi.org/10.1016/s0166-6851(00)00334-0 
Nithiuthai S, Anantaphruti MT, Waikagul J and Gajadhar A (2004). Waterborne zoonotic helminthiases. Veterinary Parasitology, 126: 167-193. DOI: https://doi.org/10.1016/j.vetpar.2004.09.018

Okamoto M, Iseto C and Shibahara T (2007). Intraspecific variation of Spirometra erinaceieuropaei and phylogenetic relationship between Spirometra and Diphyllobothrium inferred from mitochondrial CO1 gene sequences. Parasitology International, 56: 235-238. DOI: https://doi.org/10.1016/j.parint.2007.03.003

Ooi HK, Chang SL, Huang CC, Kawakami Y and Uchida A (2000). Survey of Spirometra erinaceieuropaei in frogs in Taiwan and its experimental infection in cats. Journal of Helminthology, 74: 173-176. DOI: https://doi.org/10.1017/s0022149x0000024x

Pranashinta GT, Suwanti LT, Koesdarto S and Poetranto ED (2017). Spirometra in Ptyas mucosus Snake in Sidoarjo, Indonesia. Veterinary Medicine International Conference, 1: 1-7. DOI: https://doi.org/10.18502/kls.v3i6.1104

Prasetyo RH, and Safitri E (2019). Sparganum in frog meat: A warning for the occurrence of human sparganosis. Tropical Parasitology, 9(2): 130-131. DOI: https://doi.org/10.4103/tp.TP_64_18

Qiu MH and Qiu MD (2009). Human plerocercoidosis and sparganosis. A historical review on pathology, clinics, epidemiology and control. Chinese Journal of Parasitology and Parasitic Diseases, 27: 251-260. Available at: https://www.ncbi.nlm.nih.gov

Sahoo N, Sahoo S, Mohanty B, Khuntia HK, Behera BK, and Hota SR (2018). Molecular identification of Spirometra erinaceieuropaei infection in a dog with its successful treatment. Journal of Entomology and Zoology Studies, 6(4): 1487-1489. Available at: http://www.entomoljournal.com

Scholz T, Kuchta R and Brabec J (2019). Broad tapeworms (Diphyllobothriidae), parasites of wildlife and humans: Recent progress and future challenges. Parasites and Wildlife, 9: 359-369. DOI: https://doi.org/10.1016/j.ijppaw.2019.02.001

Wang F, Zhou L and Gong S (2011). Severe infection of wild-caught snakes with Spirometra erinaceieuropaei from food markets in Guangzhou, China involves a risk for zoonotic sparganosis. Journal of Parasitology, 97: 170-171. DOI: https://doi.org/10.1645/GE-2519.1

Wiwanitkit V (2005). A review of human sparganosis in Thailand. International Journal of Infectious Disease, 9(1): 312-316. DOI: https://doi.org/10.1016/j.ijid.2004.08.003

Wei T, Zhang X, Cui J, Liu LN, Jiang P and Wang ZQ (2014). Levels of sparganum infections and phylogenetic analysis of the tapeworm Spirometra erinaceieuropaei sparganum in wild frogs from Henan Province in central China. Journal of Helminthology, 89(4): 433-438. DOI: https://doi.org/10.1017/S0022149X14000248

Wu ZJ, Chen Y and Qiu XL (2007). An investigation of plerocercoid infection of frogs in Guiyang city and analysis of clinical characteristics of 104 cases. Journal of Guiyang Medical College, 32(1): 140-141. Available at: http://en.cnki.com.cn

Yudhana A, Praja RN and Supriyatno A (2019). The medical relevance of Spirometra tapeworm infection in Indonesian bronzeback snakes (Dendrelaphis pictus): A neglected zoonotic disease. Veterinary World, 12:845-848. DOI: https://doi.org/10.14202/vetworld.2019.844-848

Zhang X, Cui J, Liu LN, Jiang P, Wang H and Qi X (2015). Genetic structure analysis of Spirometra erinaceieuropaei isolates from central and southern China. PLoS One, 10: 1371. DOI: https://doi.org/10.1371/journal.pone.0119295

Zhu XQ, Beveridge I, Berger L, Barton D and Gasser RB (2002). Single-strand conformation polymorphism-based analysis reveals genetic variation within Spirometra erinacei (Cestoda: Pseudophyllidea) from Australia. Molecular and Cellular Probes, 16: 159-165. DOI: https://doi.org/10.1006/mcpr.2001.0406 